\title{
The presence of a Bar-Like Structure in the Central Regions of Seyfert Galaxies
}

\author{
V. L. Afanasiev and A. I. Shapovalova \\ Special Astrophysical Observatory, Russian Academy of Sciences, \\ Karachai-Cherkessian Republic, 357147, Russia
}

\begin{abstract}
The central $\left(8^{\prime \prime} \times 8^{\prime \prime}\right)$ regions of the Seyfert galaxies Mrk 744 and NGC 4151 were studied by the 3D-spectroscopy method. There is evidence that a mini-bar $(0.5-2 \mathrm{kpc})$ is present in the central regions of these galaxies, which provides transportation of gas of the disk to fuel the nucleus.
\end{abstract}

\section{Observations}

Spectra of the central part of Seyfert galaxies Mrk 744 and NGC 4151 were obtained at the prime focus of the 6 - $m$ telescope with the multi-pupil field spectrograph (MPFS) and 2D photon- counting system in Trim-mode. The spectral regions were $6200-7200 \AA$ (Mrk 744) and 4600-5300 $\AA$ (NGC 4151), dispersion $1.7-1.8 \AA \mathrm{px}^{-1}$. A lens array of $9 \times 11$ (Mrk 744) or $8 \times 12$ (NGC 4151) was used, the image size from one lens corresponding to $1^{\prime \prime} 2 \times 11^{\prime \prime} 2$ (Mrk 744) or $0 " 8 \times 0.8$ (NGC 4151).

\section{Results}

\section{1. $\quad$ Mrk 744}

The Seyfert galaxy Mrk $744=$ NGC 3786 is the southern component of the pair NGC 3786/88. De Vaucouleurs classified it as type ( $\left.R^{\prime}\right) \operatorname{SAB}(\mathrm{rs})$ a. Its nucleus corresponds to type Sy1.8. The velocity field of Mrk 744 was investigated by us in 1981 (Afanasiev \& Shapovalova 1981) from the observations on the 6$\mathrm{m}$ telescope with a long slit. In the circumnuclear region $\left(3^{\prime \prime}-5^{\prime \prime}\right)$ deviations from circular motions with an amplitude of $100-150 \mathrm{~km} \mathrm{~s}^{-1}$ in position angles of $20^{\circ}-60^{\circ}$ were detected in forbidden lines. To explain this phenomenon a bar was assumed to be present in the central region of the galaxy. For this to be confirmed, we obtained spectra of the central region of Mrk744 in May 1994 with MPFS. From the spectra we made a Gauss analysis of $\mathrm{H} \alpha$, [NII] $\lambda 6584 / 48$ and [SII] $\lambda 6717 / 31$ emission line profiles. Maps of the velocity field and the brightness distributions in the emission lines (narrow components) and continuum were built. In the continuum the isophotes are elongated in the position angle $45^{\circ}$, in $\mathrm{H} \alpha$ and [NII] the inner isophotes are nearly circular, and the outer isophotes are elongated in P.A. $45^{\circ}$ in $\mathrm{H} \alpha$ and P.A. $25^{\circ}$ in [NII]. The maps of the velocity field in $\mathrm{H} \alpha$ and [NII] are different (Figure 1a, b). The 


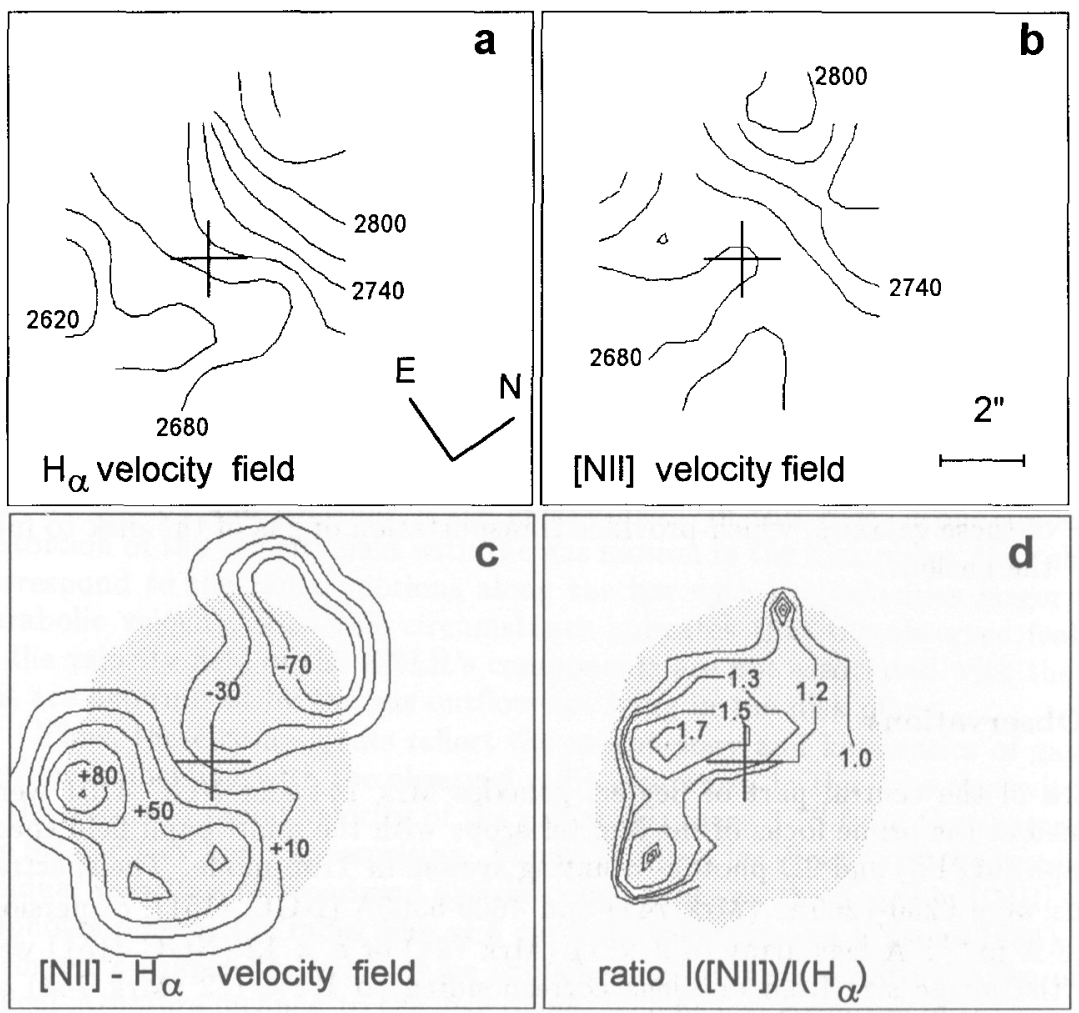

Figure 1. Kinematics of the circumnuclear region of Mrk 744 in the narrow components of emission lines $\mathrm{H} \alpha$ and [NII] $\lambda 6584$

difference in velocity field ([NII $]-H \alpha$ ) is presented in Figure 1c. At a distance of $3^{\prime \prime}-5^{\prime \prime}$ in the P.A. $25^{\circ}-45^{\circ}$ the deviations of the velocities in [NII] from $\mathrm{H} \alpha$ correspond to $-(60 \div 130) \mathrm{km} \mathrm{s}^{-1}$ (as obtained earlier from long-slit spectra), while in the opposite direction the velocity deviations amount to $+(80 \div 120)$ $\mathrm{km} \mathrm{s}^{-1}$. In Figure $1 \mathrm{~d}$ the ratios of line intensities [NII] $\lambda 6584$ to $\mathrm{H} \alpha$ (narrow component) in the form of isophotes are shown. The regions of the largest values of $\mathrm{I}([\mathrm{NII}]) / \mathrm{I}(\mathrm{H} \alpha) \sim 1.7$ are located at a distance of $3^{\prime \prime}-5^{\prime \prime}$ from the center and look like a structure close to linear, elongated in the P.A. $25^{\circ}-30^{\circ}$. We believe that the peculiarities noted above are caused by shock excitation at the borders of the bar.

\subsection{NGC 4151}

From spectra of NGC 4151 maps of the velocity field in the narrow components $\mathrm{H} \beta$ and [OIII] $\lambda 5007$ (Afanasiev \& Shapovalova 1993, Figure 2a, b), maps of the brightness distribution in these lines and in the continuum were obtained. In [OIII] the position angle of the large axis is P.A. $40^{\circ}$ and does not coincide with the direction of the radio jet (P.A. $77^{\circ}$ ). The difference in velocity field [OIII] 
- $H \beta$ has a peculiarity in velocities of $\pm 100 \mathrm{~km} \mathrm{~s}^{-1}$ at a distance of $100-150 \mathrm{pc}$ from the nucleus in the P.A. $15^{\circ}$ (Afanasiev \& Shapovalova 1994, Figure 2c). This kinematic effect is apparently associated with the presence of a gaseous mini-bar at the center of NGC 4151.

\section{Discussion}

The results of spectroscopy of Mrk 744 obtained with a long slit and MPFS at the $6-\mathrm{m}$ telescope point out to the presence of a bar-like structure $1.5-2 \mathrm{kpc}$ in size in the central part of it. On the rotation curve of Mrk 744 at a distance of about $2 \mathrm{kpc}$ from the center a local maximum is observed (Afanasiev 1981). It indicates possible presence of a bar-formation at the center ( $R \sim 0.5-2 \mathrm{kpc}$ ) of Mrk 744. The gaseous disk in the region of the bar is thinner than the latter, has lower chaotic velocities and rotates faster than the bar, $\Omega_{d}>\Omega_{b}$,. If the radius $R_{1}$, at which the relative linear velocity $\triangle V_{R}$ reaches the sound speed in the gaseous disk $\Delta V_{R}=\left(\Omega_{d}-\Omega_{b}\right) R>V_{s}$, is within the bar region $\left(R_{b}\right)$, then in region $R_{1}<R_{b}$ there may arise a shock wave. Then the shock front will be observed on the back side with respect to the direction of rotation, if the disk and the bar rotate in the same direction. Depending upon the $\triangle V_{R}$ value we shall observe either a strong or weak shock wave visible in optics (mainly in forbidden lines) as linear structures. Because of the finite thickness of the bar such linear structures will turn in the position angle with respect to the bar in the direction opposite to rotation of the bar. The bar is elongated in the P.A. $45^{\circ}$, and the peculiarities close to linear in the P.A. $25^{\circ}-30^{\circ}$ at distances $3^{\prime \prime}-5^{\prime \prime}$ from the center (Figure 1c,d) are mainly due to the component [NII] associated with shock excitation at the borders of the bar. In NGC 4151 a powerful stellar bar of $9 \mathrm{kpc}$ is also observed (Simkin 1975) in the P.A. $130^{\circ}$. We detected a peculiarity in velocities in the P.A. $15^{\circ}$ in the central part of this galaxy and assumed that this was due to the presence of a gaseous bar in the position angle $50^{\circ}$ inside the stellar bar. According to the computations done by Shlosman et al. (1990) the stellar bar may capture interstellar medium, forming a gaseous disk at scales of hundreds of parsecs approximately along the minor axis of the stellar bar, if the bar-formation is a three-axial potential.

This research has been supported by a grant from the Russian Foundation of Fundamental Research 93-02-17013.

\section{References}

Afanasiev, V. I. \& Shapovalova, A. I. 1981, Astrofizika, 17, 403 ( in Russian)

Afanasiev, V. L. 1981, Pis'ma v Astron.Zh., 7, 390 (in Russian)

Afanasiev, V. L. \& Shapovalova, A. I. 1993, in The Nearest Active Galaxies, Ed. J. Beckman et al., Madrid

Shlosman, I., Begelman, M. C., \& Frank, J. 1990, Nature, 345, 679

Simkin, S. M. 1975, ApJ, 200, 567 